\title{
On Efficient FE Simulation of Pulse Infrared Thermography for Inspection of CFRPs
}

\author{
by S. Hedayatrasa ${ }^{*, *}$, J. Segers ${ }^{*}$, J.A. Calderon Tellez ${ }^{*}$, W. Van Paepegem ${ }^{\star}$ and M. Kersemans ${ }^{\star}$ \\ * Department of Materials, Textiles and Chemical Engineering, Ghent University, Technologiepark-Zwijnaarde 903,9052 \\ Zwijnaarde, Belgium \\ ** SIM M3 program, Technologiepark 935, B-9052 Zwijnaarde, Belgium
}

\begin{abstract}
This paper reports an investigation on efficient finite element (FE) simulation of pulse infrared thermography of CFRPs. The very short duration of pulse heating as well as the layered structure and the anisotropic thermal properties of CFRPs impose specific constraints to the FE analysis of this transient thermal problem. Hence, for FE based optimization of pulse thermographic experiment, appropriate selection of FE parameters is critical so that the computational intensity is reduced without compromising the defects' quantification. Various modelling aspects including non-uniform heat flux induced by the flash lamp, element formulation and appropriate mesh sizing of out-of-plane and in-plane directions and the defect modelling approach are discussed. The effect of the modelling approach on the defect quantitative parameters and post-processing of calculated thermal images is investigated. Alternative modelling approaches are proposed and compared in terms of their reliability for defect quantification and their computational cost.
\end{abstract}

\section{Introduction}

Optical infrared thermography (IRT) has been attracting significant industrial interest to be developed as a nondestructive testing (NDT) technique with trusted reliability. An optical source radiates heat onto the surface of the test piece and an infrared camera provides full-field contactless measurement of the surface temperature evolution. This temperature evolution is interpreted for the detection and quantification of damage [1]. Lock-in and pulse thermography are the two common approaches of optical IRT. In lock-in thermography (LT) the excitation power is harmonically modulated at a particular frequency while for pulse thermography (PT) a very short heat pulse is emitted to the sample's surface with a flash lamp. PT enables excitation of a broad range of frequencies through a single excitation cycle and provides more information about the subsurface defects compared to LT, though with reduced SNR.

Simulation of IRT is of great importance as it provides support for model-based development, implementation and optimization of the experiment, and finite element FE analysis has proved its robustness in serving this purpose [2-5]. Optical IRT is based on thermal wave propagation through the depth of the test piece and its interaction with subsurface defects. Hence, the FE results should ideally be comparable with the promises of the IRT experiment and have to (i) provide fast and reliable simulation of the experiment to inspect a relatively large area, (ii) adequately define the through depth thermal behavior and (iii) provide thermal images similar to those measured by the infrared camera.

CFRPs have wide structural application and are prone to various defect types e.g. fiber breakage, matrix cracking and delamination. The strongly anisotropic thermal conductivity and multi-layer structure of CFRPs significantly affect the thermal wave propagation which should be appropriately taken into account in the FE model.

This paper reports an investigation on FE analysis of PT for inspection of CFRP composite plates with delamination type of defect. The effect of various FE modelling parameters on the accuracy and reliability of results is studied including in-plane and through-thickness mesh density, uniformity of the mesh grid and the element formulation. Different defect modelling approaches and their thermal signature are also compared. Competency of alternative FE modelling approaches in damage identification is compared and principal component analysis (PCA) [7] is implemented to evaluate the results. FE analysis is performed by ABAQUS CAE in conjunction with MATLAB for parametric study and post-processing of the results.

\section{Modeled CFRP plate and optical excitation}

A 6-plies unidirectional ([0]6) CFRP plate as shown in figure 1 (with fibers along the $x$-axis) is modelled including three $5 \mathrm{~mm}$ square delamination defects at three different depths of the laminate. Temperature dependent thermal properties are assumed for the unidirectional CFRP as measured by Joven, Das [8], with room temperature diffusivity of $\alpha_{11}=5.39 \times 10^{-6} \mathrm{~m}^{2} / \mathrm{s}$ and $\alpha_{33}=6.16 \times 10^{-7} \mathrm{~m}^{2} / \mathrm{s}$ respectively along and perpendicular to the fibers. The top surface is subjected to a pulse heating excitation as shown in figure $1(\mathrm{~b})$ and its thermal response is analyzed for evaluation of $\mathrm{PT}$ in reflection mode. ABAQUS brick elements with temperature degree of freedom and linear (8-Nodes) or quadratic (20Nodes) formulation are used, available in ABAQUS Standard. The delaminations are initially modelled by a perfect 
debonding of its adjacent plies i.e. an in-plane crack. This model results in a perfect thermal barrier for the throughthickness thermal wave propagation. Other defect modeling alternatives are explained in the Section 4.

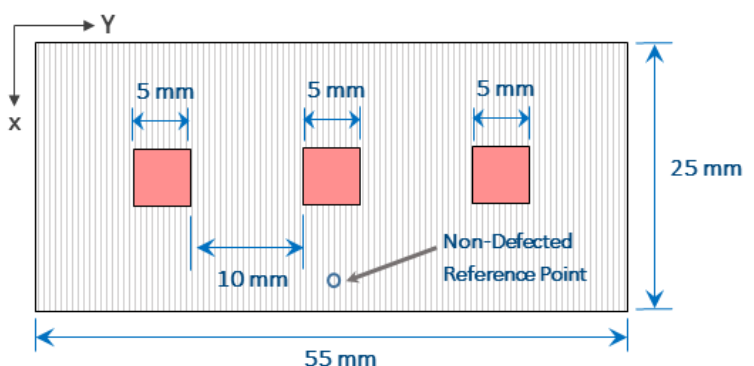

(a)

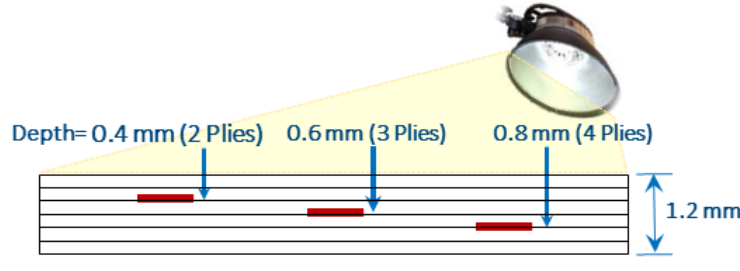

(b)

Fig. 1: Modeled unidirectional CFRP [0]6 with three in-plane defects (delaminations) of the same sizes at different depths , (a) cross-section and (b) top view of the CFRP and dimensions

The CFRP with initial room temperature of $T_{0}=25^{\circ} \mathrm{C}$ is exposed to a pulse heating. Convective and radiative thermal interactions are defined for the top and bottom surfaces of the plate which dissipate its heat energy into the surrounding medium. The assumed coefficient of heat convection and the emissivity respectively are $h=5 \mathrm{~W} / \mathrm{m}^{2} . \mathrm{k}$ and $e=0.9$. The optical source is a $Q=6 \mathrm{~kJ}$ flash lamp with beam angle of $90^{\circ}$ and pulse time of $t_{p}=10 \mathrm{~ms}$ where $25 \%$ of the lamps input energy is assumed to be effectively emitted to the CFRP's surface [9, 10]. The in-plane wave propagation induced by the non-uniform heat radiation of the lamp significantly affect the interaction of defects with thermal wave and their thermal signature. Modelling the radiative interaction of the lamp and sample, to account for this phenomena, proved to be highly computationally intensive. Therefore, a Gaussian distribution was considered for the spatial gradient of the flash lamp's heat flux as given in Eq. (1):

$$
g(x, y)=\frac{1}{2 \pi \sigma^{2}} \exp \left(-\frac{x^{2}+y^{2}}{2 \sigma^{2}}\right)
$$

where $\sigma$ is the standard deviation defining the extent of Gaussian distribution in $x y$-plane. It can be shown that $\int_{-\infty}^{+\infty} \int_{-\infty}^{+\infty} g(x, y) d x d y=1$ which 0.95 of it is included in the radius $\sqrt{x^{2}+y^{2}} \leq 2 \sigma$. Therefore, the Gaussian distribution of the lamp's heat at a radius of $r=2 \sigma$ (to include 95\% of lamp's emitted energy) over a surface with emissivity $e$ at an angle $\theta$ is:

$$
q(x, y)=\left(0.25 \frac{Q}{t_{p}} e \cos \theta\right)\left(\frac{2}{\pi r^{2}}\right) \exp \left(-\frac{2}{r^{2}}\left(x^{2}+y^{2}\right)\right)
$$

The relative position of the CFRP sample and the flash lamp and the Gaussian distribution of heat flux at a distance of $500 \mathrm{~mm}$ for $\theta=15^{\circ}$ are schematically depicted in figure 2(a) and the resulting heat flux on the CFRP sample is shown in figure $2(b)$.

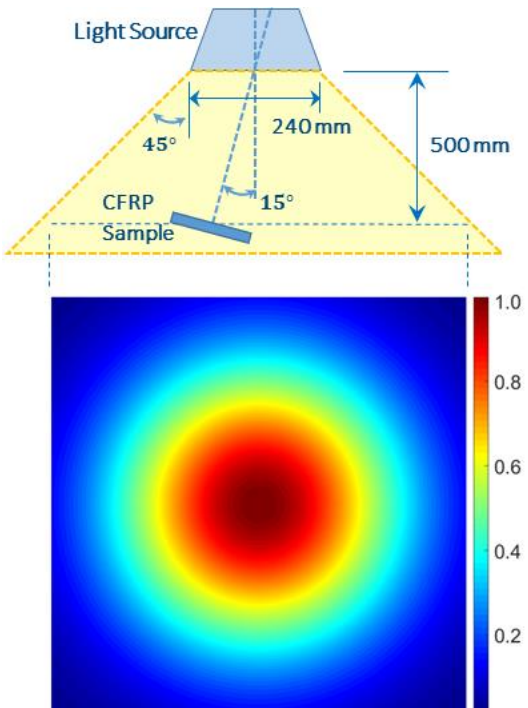

(a)

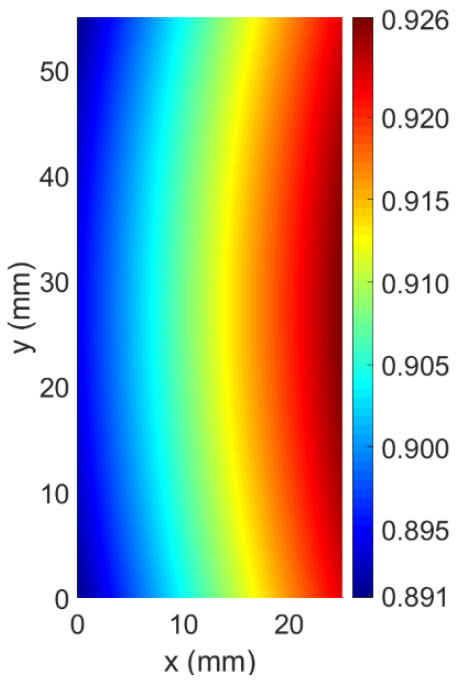

(b)

Fig. 2: Gaussian distribution of heat flux (a) at a distance of $500 \mathrm{~mm}$ from the source of radiation (assuming a beam angle of $90^{\circ}$ ) and (b) to the resulting heat flux on the CFRP sample's area (placed at $15^{*}$ angle). 


\section{FE model and quantitative defect parameters}

In this section the effect of FE mesh parameters on quantitative parameters of the defect are investigated. This allows to understand the trade-off between computational cost and reliability of FE analysis for simulation of defect quantification through PT. Temporal gradient of surface temperature and that of its second time derivative at the center of defected areas, and also their contrast with a reference non-defected point (figure 1) are selected. The temperature contrast of defected and non-defected area can reveal the defects. However, the second time derivative of the temperature provides an earlier indication of the defect and is independent of the magnitude of the out-of-plane heat flux. Of course, it is slightly affected by in-plane thermal waves induced by non-uniform heating.

\subsection{Initiating FE model}

The very short pulse of the flash lamp stimulates very high frequency out-of-plane thermal waves in the top surface of the sample which significantly increase the temperature to a very small depth corresponding to the penetration depth of those frequencies. The non-uniform heating of the lamp also induces in-plane thermal wave propagation with relatively much smaller gradients compared to out-of-plane wave. After the heating stage, the sample starts dissipating heat into the environment at a very high rate until its surface temperature is reduced. Then it gradually cools down until reaching the steady state.

Hence, for reliable and efficient modelling of this transient thermal problem it is necessary to properly select an appropriate FE mesh matching the underlying phenomena. The steady state 1D thermal wave equation results into a highly damped and dispersive thermal wave:

$$
T(x, t)=T_{0} e^{-\frac{x}{\mu}} e^{i\left(\omega t-\frac{x}{\mu}\right)}
$$

where $\omega=2 \pi f$ is the circular frequency, $\mathrm{t}$ is time and $\mu(\omega)=\sqrt{\frac{2 \alpha}{\omega}}$ is the diffusivity length and $\alpha$ the diffusivity of constitutive material. The diffusivity length $\mu$ defines the power of exponential decay as well as the wavelength of the harmonic term $\lambda=2 \pi \mu$ in spatial domain $x$. The wave amplitude at diffusivity length $\mu$, half-wavelength $0.5 \lambda$ and wavelength $\lambda$ reduces to $36.79 \%, 4.32 \%$ and $0.19 \%$, respectively. Therefore, the frequency response of interest defines the spatial gradient of temperature and as such the appropriate mesh element size.

CFRPs generally need to be modelled as a layered medium in order take into account the anisotropic thermal properties of the plies in an arbitrary lay-up. Hence the finite element model normally has at least as many element layers as the number of plies. All plies have the same out-of-plane diffusivity regardless of their orientation and the lay-up predominantly affects the in-plane wave propagation. As an initial guess, the global in-plane element size $\Delta L_{e}$ of the orthotropic model is defined as a factor of average wavelength at the highest frequency of interest for thermal analysis $\omega_{M}$ :

$$
\Delta L_{e}=\frac{\pi\left(\mu_{11}\left(\omega_{M}\right)+\mu_{22}\left(\omega_{M}\right)\right)}{n_{L}}
$$

where $\mu_{11}$ and $\mu_{22}$ are orthotropic diffusivity lengths along and perpendicular to fibres, respectively, and $n_{L}$ is an scaling factor. In this study the lowest frequency associated with the pulse duration $t_{p}$ (i.e. considering the pulse time as a half cycle) is defined as the maximum analysis frequency $\omega_{M}=\pi / t_{p}$, and a scaling factor of $n_{L}=1$ is initially assigned. The effect of out-of-plane mesh distribution, scaling factor $n_{L}$, and spatial gradient of in-plane mesh on defect quantification are investigated in the following sections.

There is a correlation between the lower limit of time increment $\Delta t_{\min }$ and the element size $\Delta L_{e}$ in the direction with the highest gradients in order to ensure that the calculated thermal behaviour is valid. This limit is determined based on the minimum time required for the heat to propagate through the element's characteristic length $\Delta L_{e}$ :

$$
\Delta t_{\min }=\frac{\Delta L_{e}^{2}}{n_{t} \alpha}
$$

where according to ABAQUS documentation a value of $n_{t}=6$ is recommended. For a material like CFRP which has relatively low out-of-plane diffusivity (through which highest thermal gradients occurs) this lower limit of time increment is quite large compared to the pulse time. Therefore, very small elements are required to adequately simulate the thermal gradients of top surface during pulse heating and immediately after it (i.e. for evaluation of shallower defects with early thermal indications). The rate of thermal gradients significantly reduces from beginning of heating until later stages of cooling down, and it is best to adopt a gradient FE mesh for highest computational efficiency as discussed in the following section.

\subsection{Through-thickness FE mesh}

The top ply of the model is exposed to the pulse heating and experiences extremely high temperature gradient during the heating stage and a short time immediately after it. At the lowest frequency corresponding to the pulse time $0.5 / t_{p}=50 \mathrm{~Hz}$ the out-of-plane wavelength in CFRP model is $0.393 \mathrm{~mm}$ which at half of this wavelength (i.e. less than a ply thickness) the wave's amplitude reduces to just $4.32 \%$. An element with quadratic formulation may capably define this 
thermal gradient. In case of using linear elements it is advised to enhance the out-of-plane model of the top ply with a graded multi-layer mesh as shown in the Figure 3 in which the element size biasedly reduces from bottom to top.

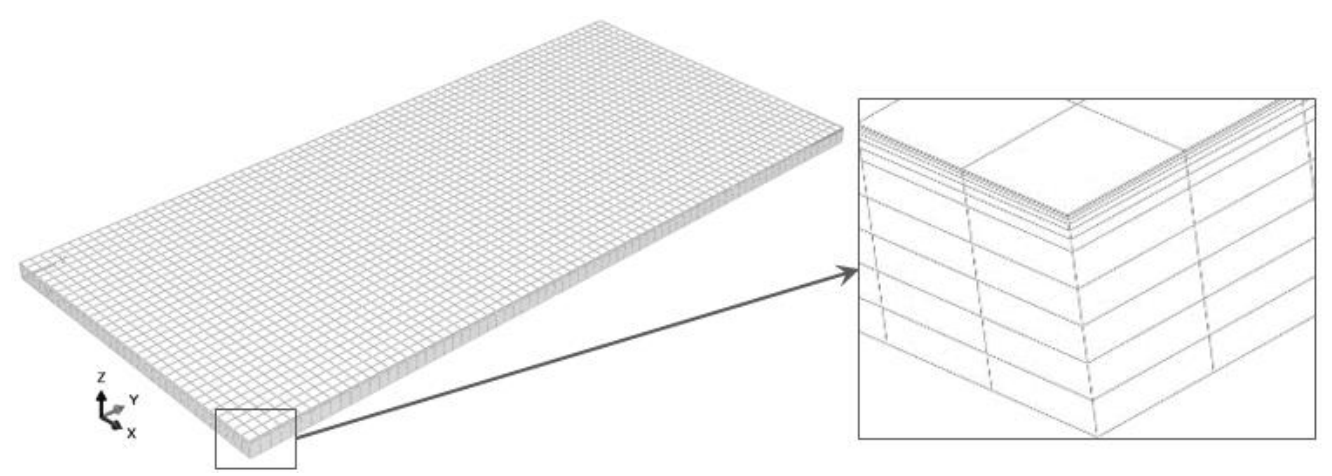

Fig. 3: FE model and graded mesh of the top ply

To study the effect the top ply's mesh density, the CFRP is modelled by linear and also quadratic elements, and the top ply is modelled with 1 to 10 layers of elements in linear model and with 1 to 4 layers of element layers in quadratic model, all with biased graded distribution. No noticeable improvement was observed for having more than 6-layers of linear elements and more than 2-layers of quadratic elements. According to figure 4(a) the calculated surface temperature at the center of a defected area vitally depends on the FE mesh of the top ply. Although linear and quadratic FE models seem to converge in terms of magnitude of temperature, their difference in terms of second time derivative of temperature is shown in figure 4(b).

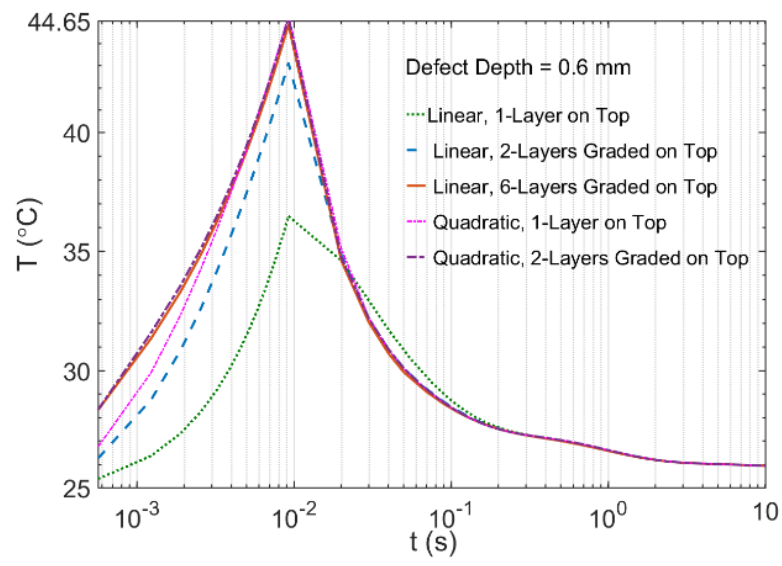

(a)

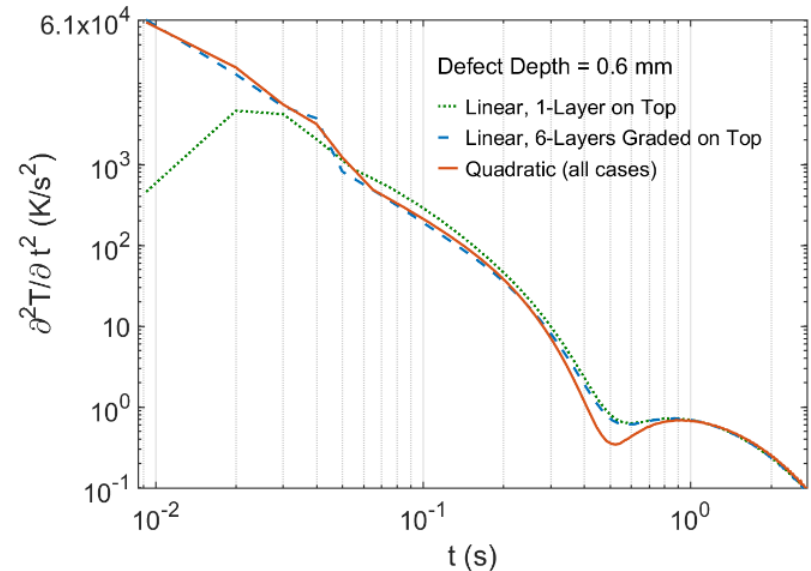

(b)

Fig. 4: Thermal response at the centre of the defect at depth $0.6 \mathrm{~mm}$ for various FE meshes, (a) temperature and (b) $2^{\text {nd }}$ time derivative of temperature versus time; $\left(n_{L}=1\right)$

In practice, the measurement during the heating flash is not generally used because it is too short to reveal a subsurface defect of interest, and it is actually not measurable due to very high intensity of flash radiation and saturation of the infrared camera. Moreover, the existence of a defect may be realized due to the difference of thermal response at a defected and non-defected area. Therefore, the contrast of surface temperature and its second time derivative at the center of defected areas and a reference non-defected area $T_{s}$ (see figure 1) are further studied during cooling the stage only as shown in figure 5 .

Figure 5(a) demonstrates the difference of temperature contrast for the three defects and their sensitivity to FE model formulation and the top ply's mesh density. The quadratic model leads to initially lower temperature contrast right after the heating stage and slightly higher temperature contrast once the thermal wave reflected from the defect reaches the top surface. Regarding the second time derivative contrast as shown in figure 5(b) the linear and quadratic model have good agreement except of their deviation at the local minimum which is simply caused by the time resolution of the FE solution. Other than the top ply, the mesh resolution of the lower plies also contributes to the accuracy of the calculated temperature on the top surface. According to the thermal wave theory (Eq. (1)), the deeper the ply is, the lower frequency response and therefore less out-of-plane temperature gradients it experiences. 


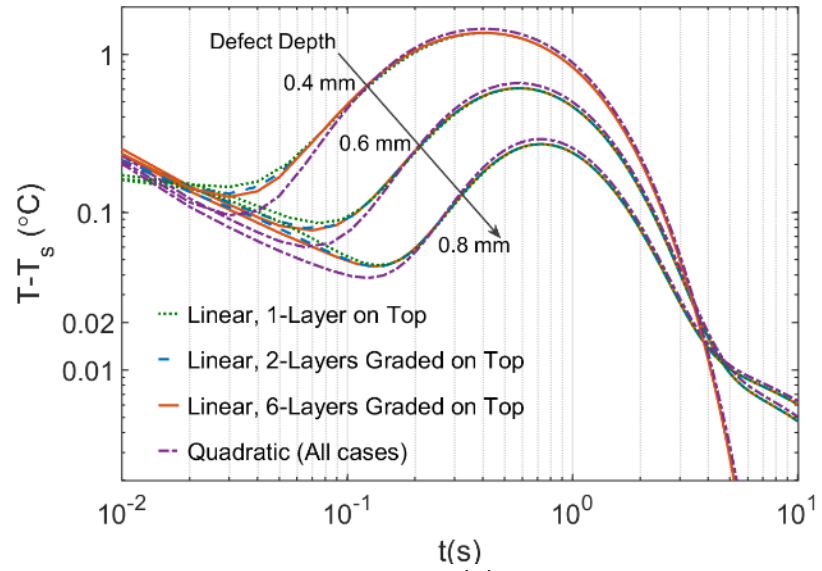

(a)

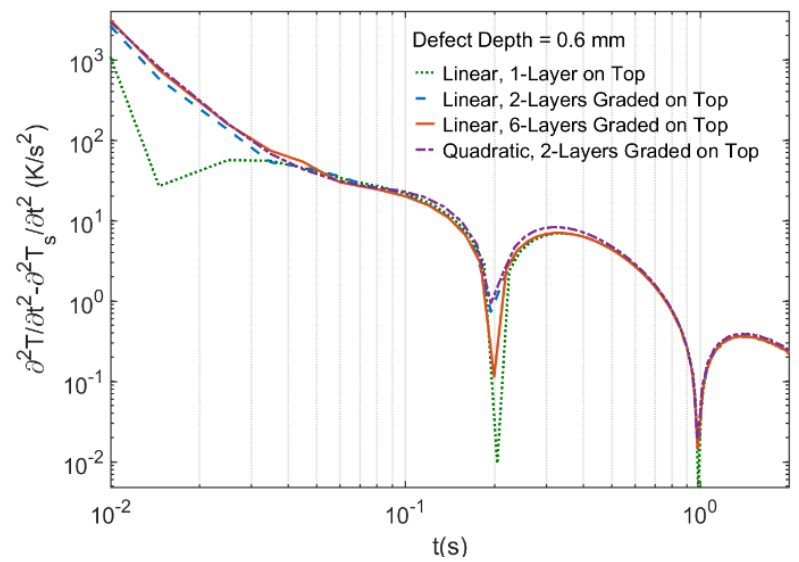

(b)

Fig. 5: Thermal response at the centre of defected area for various element layers in the top ply, (a) temperature and (b) $2^{\text {nd }}$ time derivative of temperature versus time; $\left(n_{L}=1\right)$

As evidenced by the results shown in figure 6, having more than one layer of linear elements in lower plies slightly changes the contrast of temperature and its second time derivative. This deviation reduces by the defect's depth. However, the linear and quadratic models do not converge yet due to the fact that quadratic formulation also affects the in-plane wave propagation phenomena and thermal response of the non-defected reference point.

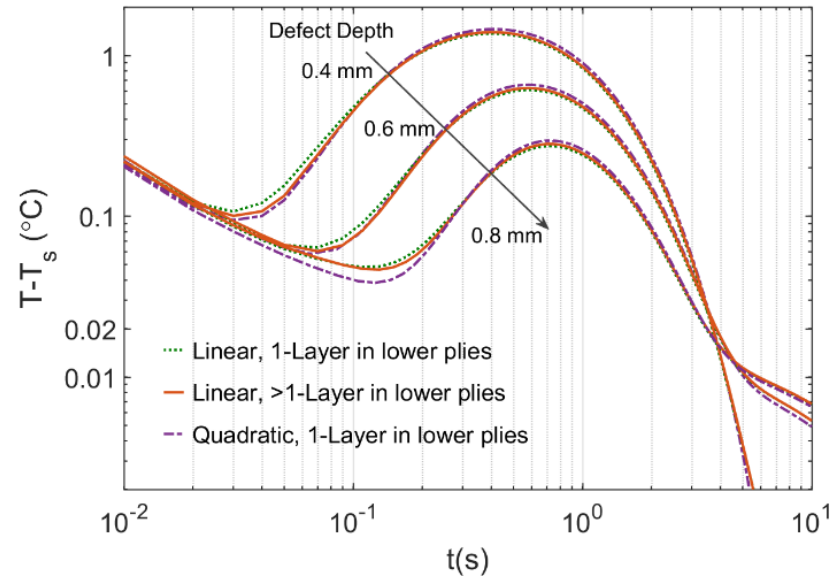

(a) $\mathrm{T}$

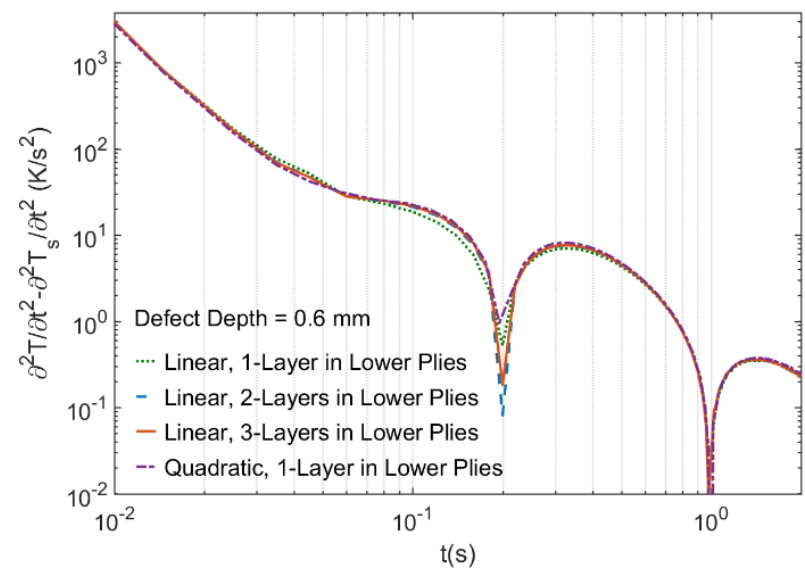

Log d

Fig. 6: Thermal response at the centre of defected area for various number of element layers in lower plies, (a) temperature contrast and (b) $2^{\text {nd }}$ time derivative of temperature; in all cases the top ply is modelled by a graded mesh (6-layers linear or 2-layers quadratic); $\left(n_{L}=1\right)$

Overall, the linear model with 6-layers of graded mesh in the top ply and single layer for other plies provides reliable results for a computational time of 10.16 minutes, compared with the quadratic model with 2-layers in top ply which has an almost double computational time of 23.41 minutes (Intel Core i7-7820HQ CPU @ $2.90 \mathrm{GHz}$, RAM 32 GB). One may consider 2 element layers for the lower plies of linear FE model which increases the computational time of current model to 14.19 minutes. However, for thicker laminates with numerous plies, it is sufficient to consider more than one element layer only for a few top most plies which contribute in the high frequency thermal response.

\subsection{In-plane FE mesh}

Other than out-of-plane mesh density, the in-plane mesh density of the FE model should also be defined such that the in-plane wave propagation induced by non-uniform heating and in-plane diffusion of reflection from the defects can be reliably calculated. In this section the implemented formulation (Eq.(4)) is examined with three different scaling factors of $n_{L}=0.5,1.0$ and 2.0. According to the contrast results shown in figure 7, the coarsest in-plane mesh corresponding to $n_{L}=0.5$ shows a significant deviation while the denser meshes corresponding to $n_{L}=1.0$ and 2.0 show a quite good agreement particularly in terms of contrast in the second time derivative of temperature (see figure 7(b)). Regarding the contrast of temperature itself, the deepest defect indicates a deviation of results by $n_{L}=1.0$ and 2.0 . This can be justified by the fact that the deeper the defects is, the more it is affected by the 3D heat diffusion. However, the 
results of the quadratic FE model with $n_{L}=1.0$ still have slight deviation from the linear FE model at the onset of rise of temperature contrast induced by the defects.

A noteworthy subject concerning the in-plane mesh of FE model for simulation of PT, is the distribution of nodes on the top plane exposed to the heat flux. If linear elements with lumped heat capacity approximation are used, then a non-uniform grid of nodes leads to spurious spatial temperature fluctuations as shown in figure 8(a). The nodes surrounded by bigger elements have higher lumped capacity and therefore lower temperature rise, and vice versa. This error provides misleading spatial gradient of temperature particularly in heating stage and immediately after it during which the nodal temperature is dominantly determined by the heat flux in the absence of heat reflection from defects. Increasing the time increment of transient analysis or using extremely small elements may fix this issue, which the first approach is limited by the very short duration of pulse and the second approach imposes computational cost.

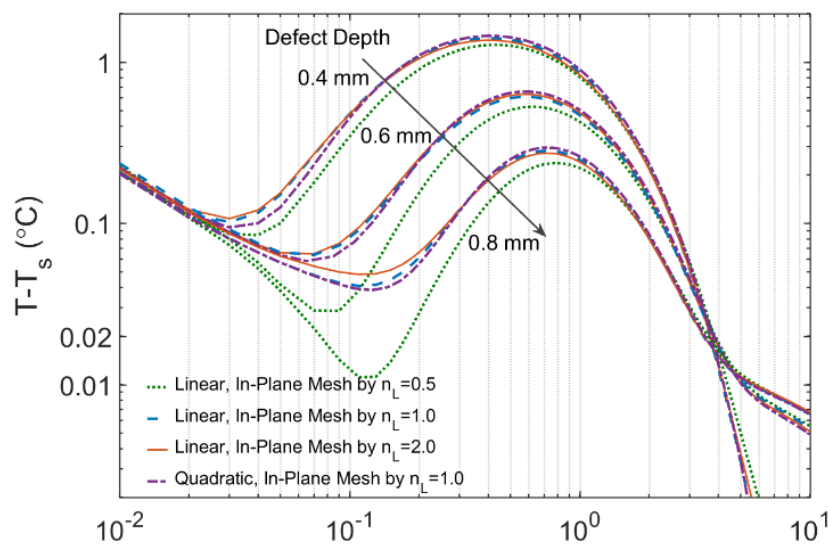

(a)

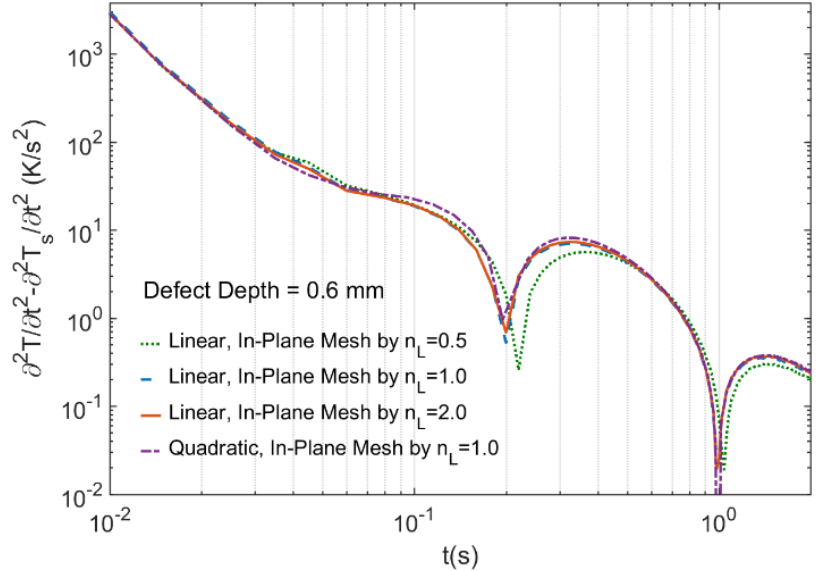

(b)

Fig. 7: Thermal response at the centre of defected area for various in-plane meshes, (a) temperature contrast and (b) $2^{\text {nd }}$ time derivative of temperature; in all cases the top ply is modelled by graded mesh (6-layers linear or 2-layers quadratic);( $\left.n_{L}=1\right)$

However, as demonstrated in figure 8(b) and (c), there are other two more alternatives: (i) use of quadratic elements in case of requiring non-uniform mesh or (ii) use of linear elements if a uniformly structured mesh is possible. Although, the CFRP sample studied herein can be simply modelled by a structured mesh, it is generally not possible when components with arbitrary geometry are to be modelled. A fully quadratic model is costly, and only the topmost ply of the CFRP is subjected to the heating pulse and experiences the very high temperature gradients in the FE model. Hence, a computationally efficient approach for FE modelling with free non-uniform mesh is to use quadratic elements only in the top ply.

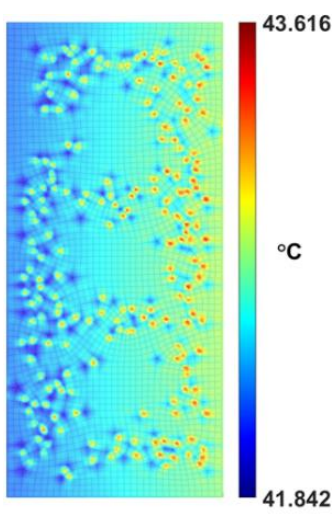

(a)

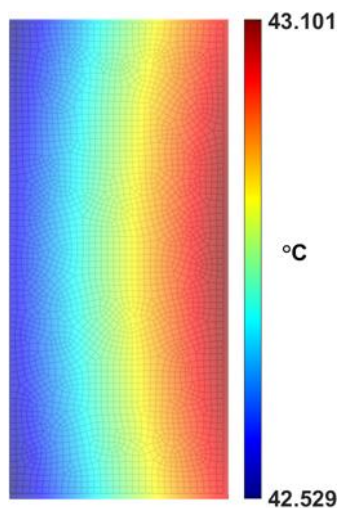

(b)

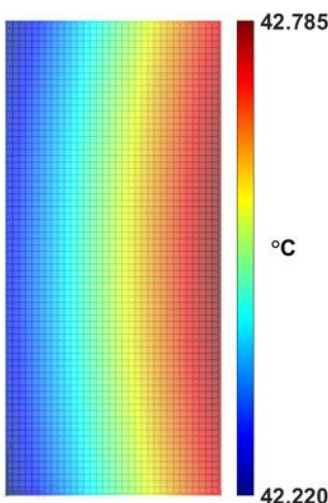

(c)

Fig. 8: Spatial gradient of temperature at the end of heating pulse $t=10 \mathrm{~ms}$ for various FE mesh types, (a) non-uniform (free) mesh of linear elements, (b) non-uniform mesh of quadratic elements and (c) uniform (structured) mesh of linear elements ; $\left.n_{L}=1\right)$

In order to assess the influence of element formulation on the thermal response of the top surface and on the damage quantification, three different FE models with free non-uniform mesh are evaluated by PCA [7] of the cooling stage. PCA determines the principal independent behavior of the response by defining the orthogonal vectors of measured data on a statistical basis. The modelling parameters considered for the three alternative FE modelling approaches are given table 1 , and the relevant PCA results are depicted in figure 9. 
Table 1. FE modelling alternatives with non-uniform in-plane mesh density and $n_{L}=1.0$

\begin{tabular}{|c|c|c|c|c|c|}
\hline \multirow{2}{*}{ Abbreviation } & \multicolumn{2}{|c|}{ Top ply elements } & \multicolumn{2}{c|}{ Lower plies elements } & \multirow{2}{*}{$\begin{array}{c}\text { Run time } \\
\text { (min) }\end{array}$} \\
\cline { 2 - 5 } & Formulation & Element layers & Formulation & Element layers & 23.11 \\
\hline MA1 & Quadratic & 2-Layers Graded & Quadratic & 1 & 11.18 \\
\hline MA2 & Quadratic & 2-Layers Graded & Linear & 1 & 10.89 \\
\hline MA3 & Linear & 6-Layers Graded & Linear & 1 & 1 \\
\hline
\end{tabular}

* Intel Core i7-7820HQ CPU @ 2.90 GHz, RAM 32 GB

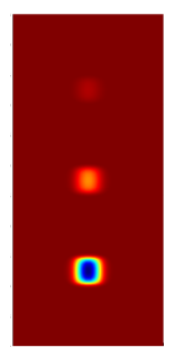

PC1

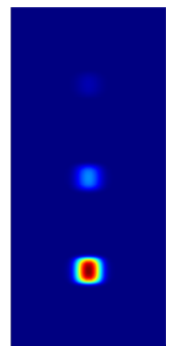

PC1

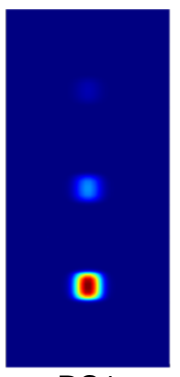

PC1

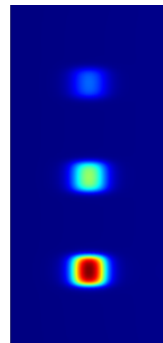

PC2

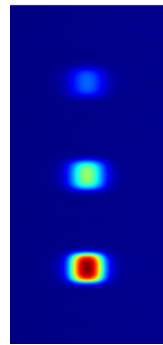

PC2

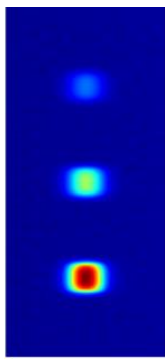

PC2

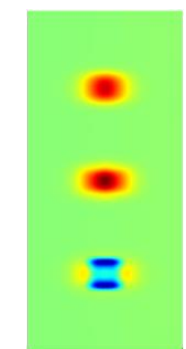

PC3

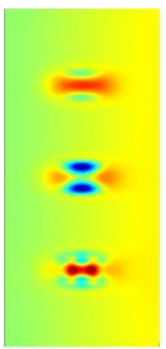

PC4

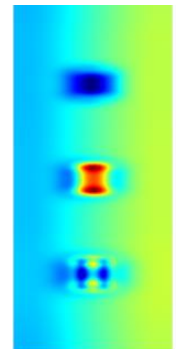

PC5

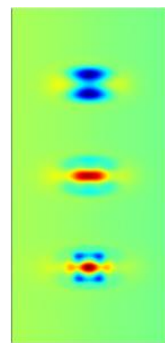

PC6

(a) PCA of results by MA1 (see table 1 )

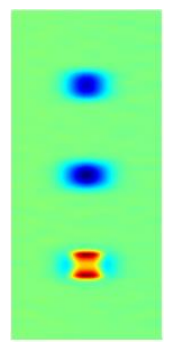

PC3

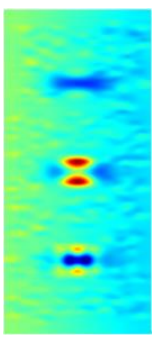

PC4

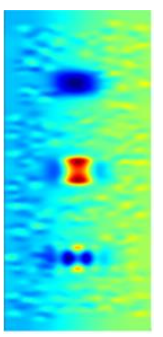

PC5

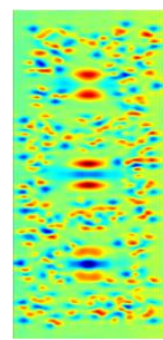

PC6

(b) PCA of results by MA2 (see table 1 )

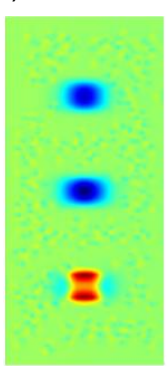

PC3

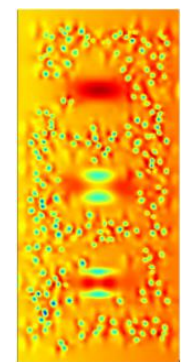

PC4

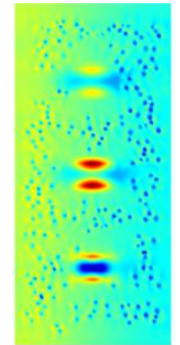

PC5

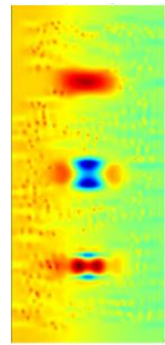

PC6

(c) PCA of results by MA3 (see table 1)

Fig. 9: Influence of FE modelling approach (with non-uniform in-plane mesh) on PCA of the thermal response calculated for a CFRP sample with in-plane crack type defect

The PCA results shown in figure 9 clearly demonstrate the difference of spatial gradient of temperature on the top surface for the different FE modeling approaches. The fundamental principal components PC-1 to PC-3 are almost the same regardless of the minor fluctuations visible in the non-defected area of PC-3 corresponding to the MA3 with nonuniform mesh of linear elements. The fully quadratic FE model MA1 has smooth high order principal components, the top quadratic model MA2 improves PC4 and PC5 with slightly added computational cost compared to the fully linear model MA3 which has significantly degraded PC4 to PC6 with intense localized fluctuations.

Consequently, the top quadratic model MA2 can be a computationally efficient and reliable approach for FE modelling with non-uniform in-plane mesh density. It is noteworthy that in practice, the noise level of thermal images taken by the infrared camera already degrades the spatial and temporal fluctuation of measured values which may alleviate the degrading effect of non-uniform mesh density. This depends on the noise level of the camera for which a PT experiment is to be simulated and also the signal to noise ratio of defects according to their depth and thermal resistance. 


\section{Thermal resistance of a delamination and post-processing of its noisy FE thermal response}

So far in this paper, the delamination was modeled by a perfect thermal barrier through an in-plane crack which assigns disconnected node pairs to the plane of defected area between the two adjacent plies of the delamination. However, in reality the delamination may be an air-filled gap between two plies or, as in common research practice, mimicked by e.g. a PTFE (teflon) insert. Here, we consider a thickness of $13 \mu \mathrm{m}$ for both. This value is chosen as it is the advised thickness to represent a delamination with a teflon insert [11].

Figure 10 compares the thermal response of delaminations modeled as (i) an in-plane crack, (ii) an air-filled gap of $13 \mu \mathrm{m}$ and (iii) a teflon insert having the same thickness of $13 \mu \mathrm{m}$ [11]. Thermal conductivity of $0.024 \mathrm{~W} / \mathrm{m}$. K, constant volume heat capacity of $718 \mathrm{~J} / \mathrm{kg}$. K and density of $1.225 \mathrm{~kg} / \mathrm{m}^{3}$ is considered for air at room temperature, and thermal conductivity of $0.25 \mathrm{~W} / \mathrm{m}$. K, heat capacity of $1050 \mathrm{~J} / \mathrm{kg}$. K and density of $2200 \mathrm{~kg} / \mathrm{m}^{3}$ for the teflon.

Figure 10(a) shows the surface temperature rise at the center of the defects and figure 10(b) shows the temperature contrast with the non-defected reference point. As the state of the art cooled infrared cameras have noise equivalent differential temperature (NEDT) of less than $20 \mathrm{mK}$, this level is indicated in the contrast data of figure 10(b) to highlight the portion of defect's thermal signature which is expected to be measured by such a camera. Evidently, the crack leads to highest temperature rise and contrast, while the teflon insert shows the lowest one.

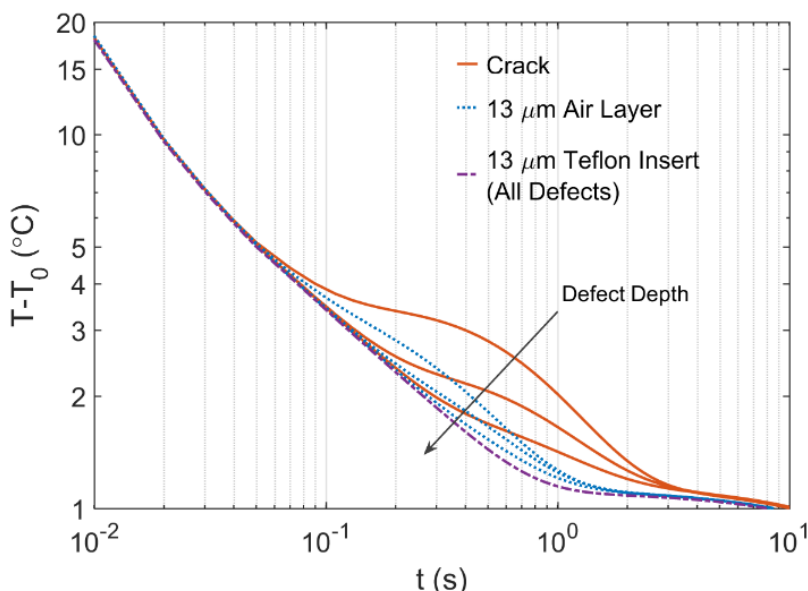

(a)

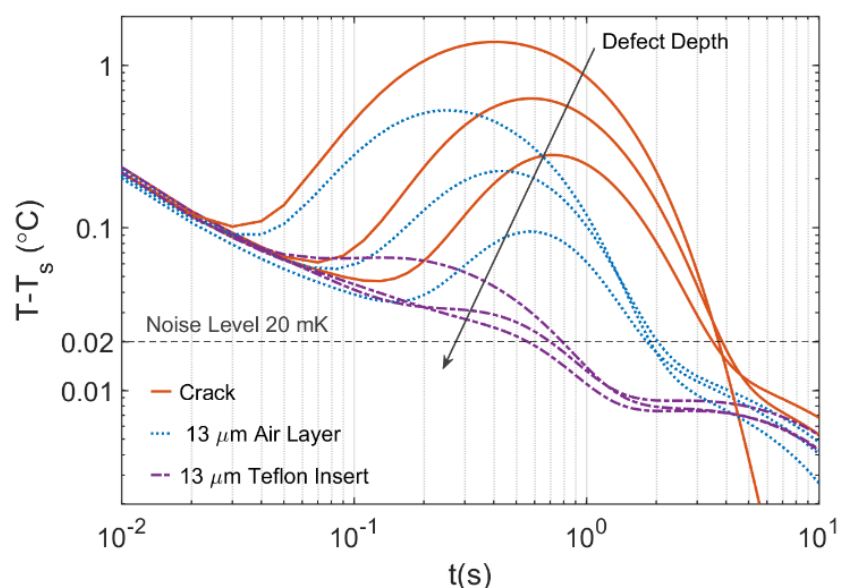

(b)

Fig. 10: Thermal response at the center of various defect types, (a) temperature rise with respect to initial temperature $T_{0}=25^{\circ} \mathrm{C}$ and (b) temperature contrast with respect to temperature of a non-defected point $T_{S}$ as shown in figure 1

(Note: the samples with $13 \mu \mathrm{m}$ defects, have increased thickness of $3 \times 13=39 \mu \mathrm{m}$ equivalent to $3.25 \%$ )

\section{5}

\section{Conclusion}

FE simulation of PT for inspection of CFRPs with delamination type defects was investigated. The effect of various FE parameters on the accuracy and reliability of the results were studied including in-plane and through-thickness mesh density, uniformity of the mesh grid and the element formulation. Different defect modelling approaches and their thermal signature were also compared.

A graded mesh in the top ply of CFRP was proposed to accurately model the heating of top surface during a short pulse. 6-layers of linear elements and 2-layers of quadratic elements both proved to be adequate for modelling a $10 \mathrm{~ms}$ pulse on a CFRP laminate with a ply thickness of $0.2 \mathrm{~mm}$. Although the thermal response of a non-graded mesh converges to that of graded mesh shortly after cooling stage starts, this part distorts the thermal response of areas with shallower defects and affects their quantitate evaluation. Moreover, it is vital to understand the maximum temperature rise in specific sample in order to avoid its degradation by excess heat.

Linear elements with lumped heat capacity approximation showed high sensitivity to the non-uniform in-plane mesh density an led to erroneous spatial fluctuation of temperature on the top surface. FE modelling alternatives were recommended and evaluated, among which, a model with quadratic element formulation only in the top ply improved the results with slightly higher computational cost compared to a fully linear model. However, for defects with low thermal contrast (i.e. those with low thermal resistance or deep ones) a fully quadratic FE model is essential so that the trace of defects thermal contrast is not faded out by spurious temperature fluctuations induced by linear elements of lower plies.

The delamination was modeled by an in-plane crack (i.e. perfect debonding of plies), an air-filled gap and also, as in common research practice, by a teflon insert. The thermal contrasts calculated for these three different modelled delaminations were quite different as expected from their significant difference in thermal resistance. Therefore, for FE evaluation of PT of delaminated CFRPs, appropriate selection of defect modelling is essential to avoid underestimation or overestimation of this NDT technique. 
Acknowledgments: The work leading to this publication has been funded by the SBO project DETECT-IV (Grant no. 160455), which fits in the Macro-ModelMat (M3) research program funded by SIM (Strategic Initiative Materials in Flanders) and VLAIO (Flanders Innovation \& Entrepreneurship Agency).

\section{References}

1. Maldague, X.P., Nondestructive evaluation of materials by infrared thermography. 2012: Springer Science \& Business Media.

2. Rizi, A.S., et al., FEM modeling of ultrasonic vibrothermography of a damaged plate and qualitative study of heating mechanisms. Infrared Physics \& Technology, 2013. 61: p. 101-110.

3. Oswald-Tranta, B., Time and frequency behaviour in TSR and PPT evaluation for flash thermography. Quantitative InfraRed Thermography Journal, 2017: p. 1-21.

4. Grys, S., L. Vokorokos, and L. Borowik, Size determination of subsurface defect by active thermography Simulation research. Infrared Physics \& Technology, 2014. 62: p. 147-153.

5. Peeters, J., et al., Robust quantitative depth estimation on CFRP samples using active thermography inspection and numerical simulation updating. NDT \& E International, 2017. 87: p. 119-123.

6. $\quad$ Shepard, S.M., et al. Enhancement and reconstruction of thermographic NDT data. in Proc. SPIE. 2002.

7. Rajic, N., Principal component thermography for flaw contrast enhancement and flaw depth characterisation in composite structures. Composite Structures, 2002. 58(4): p. 521-528.

8. Joven, R., et al., Thermal Properties of Carbon Fiber-Epoxy Composites With Different Fabric Weaves. SAMPE, Charleston, SC, 2012.

9. Shepard, S., J. Lhota, and T. Ahmed, Flash thermography contrast model based on IR camera noise characteristics. Nondestructive Testing and Evaluation, 2007. 22(2-3): p. 113-126.

10. Krankenhagen, R. and C. Maierhofer, Measurement of the radiative energy output of flash lamps by means of thermal thin probes. Infrared Physics \& Technology, 2014. 67: p. 363-370.

11. AC09036782, A., Standard test method for mode I interlaminar fracture toughness of unidirectional fiberreinforced polymer matrix composites. 2007: ASTM Internat. 